information. ${ }^{2}{ }^{3}$ The incorporation of IdU by tumour cells during S-phase can be measured by immunostaining. This method is more accurate than simple quantification of DNA by FACS analysis and avoids the use of $\left[{ }^{3} \mathrm{H}\right]-$ labelled thymidine. We have studied the reliability of assessing the S-phase fraction in FNAs by in vitro labelling with IdU as a more practical alternative to in vivo labelling. Both methods have disadvantages. In vivo, IdU may not penetrate the tumour completely and intra-tumoral concentrations of IdU vary from patient to patient, adversely affecting the sensitivity of the measurement. In vitro, aspiration may result in cell death, thereby giving a lower S-phase fraction. To control for these potential problems, we also measured the $S$-phase fraction in tumour sections after the in vivo administration of IdU.

The S-phase fraction was measured using all three methods in 11 of the 17 patients included in the study. In one patient the S-phase fraction measured in one of the FNAs was far too low. This was because most cells in the sample were dead. In the other 10 patients, however, no systematic increase or decrease in the S-phase fraction was noted when FNAs and frozen sections were compared. The results obtained with in vitro labelling are generally equivalent to those obtained with in vivo labelling. This suggests that administration of IdU to patients can be avoided.

The S-phase fraction in human breast cancer is generally low, the median value varying between 3.5 and 7.5..$^{3-6}$ Because of this low figure and the variation caused by the labelling methods, analysis of FNAs after in vitro or in vivo labelling with IdU is not suitable for monitoring response to treatment.

1 Fernando IN, Titley JC, Powles TJ, Dowsett M, Trott PA, Ashley SE, et al. Measurement of S-phase fraction and ploidy in sequential fine-needle aspirates from primary human breast tumours treated with tamoxifen. $\mathrm{Br} \mathcal{F}$ Cancer 1994;70:1211-16.

2 Remvikos Y, Beuzeboc P, Zajdela A, Voillemot N, Magdelenat $\mathrm{H}$, Pouillart P. Correlation of pretreatment proliferative activity of breast cancer with the response to cytotoxic chemotherapy. F Natl Cancer Inst 1989;81:1383-7.

3 Gaglia P, Bernardi A, Venesio T, Caldarola B, Lauro D, Cappa APM, et al. Cell proliferation of breast cancer evaluated by anti-brdu and anti-Ki- 67 antibodies: its prognostic value on short-term recurrences. Eur $\mathcal{f}$ Cancer 1993;29A: 1509-13.

4 Rew DA, Campbell ID, Taylor I, Wilson GD. Proliferation indices of invasive breast carcinomas after in vivo 5-bromo-2-deoxyuridine labelling: a flow cytometric study of 75 tumours. Br f Surg 1992;79:335-9.

5 Merlo GR, Venesio T, Bernardi A, Canale L, Gaglia P, Lauro D, et al. Loss of heterozygosity on chromosome 17 p13 in breast carcinomas identifies tumors with high proliferation index. Am F Pathol 1992;140:215-23.

6 Meyer JS, Koehm SL, Hughes JM, Higa E, Wittliff JL, Lagos JA, et al. Bromodeoxyuridine labeling for S-phase measurement in breast carcinoma. Cancer 1993;71:3531-40.

\title{
Sclerosing lymphocytic lobulitis in the male breast
}

\author{
A H S Lee, B Zafrani, G Kafiri, S Rozan, R R Millis
}

\section{Imperial Cancer Research Fund Clinical Oncology Unit, Guy's Hospital, London \\ A H S Lee \\ R R Millis}

\section{Section Medicale et} Hospitaliere, Institut Curie, Paris, France

B Zafrani

S Rozan

\section{Histopathology}

Department,

Hippocration General

Hospital,

Athens, Greece

G Kafiri

Correspondence to: Dr A H S Lee, University Department of Pathology,

Mailpoint 813,

Level E, South Block

Southampton General

Hospital,

Southampton SO16 6YD.

Accepted for publication 20 February 1996

\begin{abstract}
Sclerosing lymphocytic lobulitis is an inflammatory disorder of the breast that is well recognised in women. It has only been reported previously in two men; two further men with the condition are described here. Both presented with a breast mass, and one was an insulin dependent diabetic. Biopsy specimens from both patients showed circumscribed perivascular and, to a lesser extent, periductal collections of B and T lymphocytes. Sclerosing lymphocytic lobulitis in the female breast shows predominantly perilobular inflammation. The predominantly perivascular distribution in men is consistent with the relative paucity of epithelium in the male breast. Interlobular fibrosis with epithelioid fibroblasts was also present. (f Clin Pathol 1996;49:609-611)
\end{abstract}

Keywords: breast, male, sclerosing lymphocytic lobulitis, inflammation.

Sclerosing lymphocytic lobulitis is a recently recognised disorder of the breast characterised by perilobular and perivascular aggregates of $B$ and $\mathrm{T}$ lymphocytes, with increased expression of class II major histocompatibility antigens by the lobular and ductal epithelium, fibrosis and lobular atrophy. ${ }^{12}$ It is thought to be of autoimmune aetiology and is associated with other autoimmune diseases, particularly diabetes mellitus. The condition is well recognised in women, but has been reported in only two men. ${ }^{3}$ We describe two additional male patients in whom the perivascular and periductal inflammation has been characterised immunohistochemically.

\section{Case reports}

Patient 1 was a 47 year old Algerian man who presented with a $15 \mathrm{~mm}$ mass in the right breast. He had had insulin dependent diabetes mellitus for 31 years, but no history of other autoimmune diseases. Patient 2 was a 53 year old Greek man who presented with a poorly defined $5 \mathrm{~cm}$ breast mass. He did not have diabetes mellitus nor any other autoimmune disease, nor a family history of diabetes.

PATHOLOGICAL FINDINGS

An excision biopsy specimen was taken of the breast mass of both men. Sections from both 


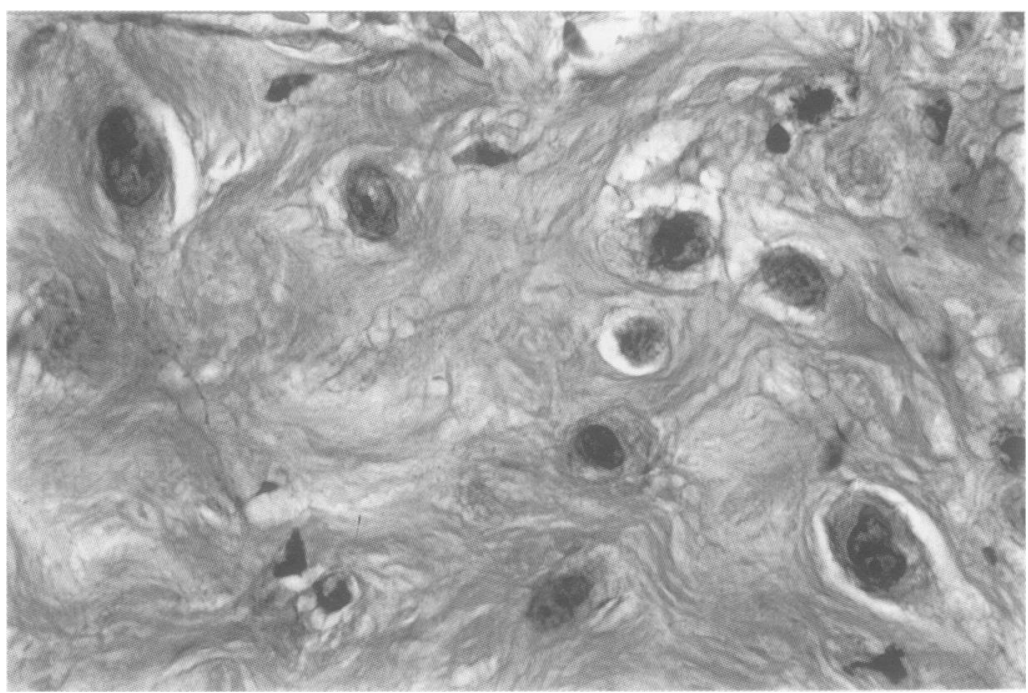

Figure 1 Plump fibroblasts were prominent in the stroma in patient 1.

showed male breast with no lobules. In patient 2 there was some epithelial hyperplasia and loose cellular periductal stroma consistent with gynaecomastia. In both biopsy specimens the interductal stroma showed fibrosis with plump fibroblasts with large oval nuclei that had an irregular outline and contained one or two small nucleoli. These fibroblasts were incon-
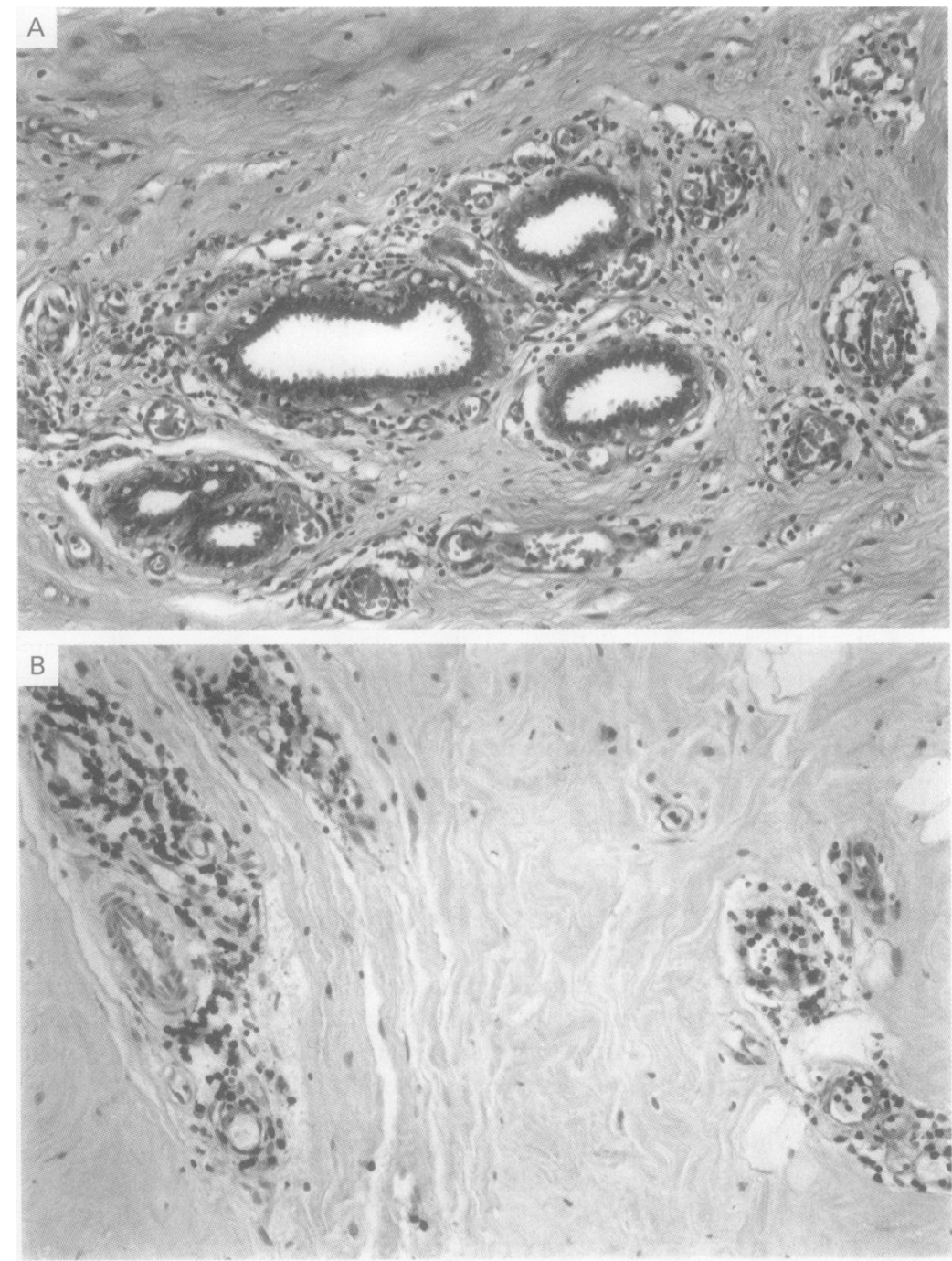

Figure 2 A circumscribed lymphocytic infiltrate is present $(A)$ around ducts and $(B)$ around blood vessels. spicuous in patient 2 and prominent in patient 1 (fig 1). In both there was a circumscribed, well defined inflammatory infiltrate around blood vessels and, to a lesser extent, around ducts (fig 2).

The infiltrate was characterised by immunohistochemistry on paraffin wax sections using a Streptavidin biotin technique and the following primary antibodies: CD3 ( $\mathrm{T}$ cells, 30 minutes microwave pretreatment; Dako, High Wycombe, UK); UCHL1 (CD45RO, primed T helper cells, macrophages, and a subset of $B$ cells; Professor PCL Beverley, ICRF, London); L26 (CD20, B cells, 15 minutes microwave pretreatment; Dako); PGM1 (CD68, macrophages, 10 minutes trypsin; Dako); TAL-1B5 ( $\alpha$ chain of HLA-DR, 15 minutes microwave pretreatment; ICRF, London ${ }^{5}$ ).

The perivascular and periductal inflammation was composed of lymphocytes, with a slight predominance of $\mathrm{T}$ cells over $\mathrm{B}$ cells in patient 1 , and predominance of $B$ cells in patient 2. There were few macrophages. In each there was patchy staining of the ductal epithelium for HLA-DR.

\section{Discussion}

Sclerosing lymphocytic lobulitis is well recognised in women, but we have found only two previous reports of the condition in men. Both had longstanding insulin dependant diabetes mellitus and presented with a breast mass. Ashton et $a l^{4}$ emphasised the prominent lymphocytic vasculitis and less noticeable periductal lymphocytic infiltrate; Tomaszewski et $a l^{\beta}$ made no distinction between the changes seen in men and women. The inflammatory infiltrate was not characterised in the former report. In the latter the inflammation was composed predominantly of $B$ cells, and there was negligible staining for HLA-DR with LN3. Both reports emphasised the presence of epithelioid fibroblasts in the fibrosis.

In both of our patients there was a perivascular and periductal lymphocytic infiltrate in a pattern quite similar to sclerosing lymphocytic lobulitis in the female breast, except that in women there is perilobular rather than periductal inflammation. The predominantly perivascular distribution in men is consistent with the relative paucity of epithelium in the male breast. Although the inflammation was around the vessels, there was no vessel damage, thus it is not a vasculitis as suggested in previous reports. ${ }^{4}$ Plump fibroblasts were a prominent feature in patient 1 , a diabetic man. It has been suggested that these fibroblasts are a marker of diabetic mastopathy, ${ }^{3}$ but they have also been reported in lymphocytic lobulitis not associated with diabetes. ${ }^{4}$ We have seen plump fibroblasts frequently in mammary stroma, and while they may be helpful in recognising sclerosing lymphocytic lobulitis, in our experience they are not a discriminatory feature. Although there was a slight predominance of $\mathrm{T}$ cells in one of our cases, B cells were prominent in both, a characteristic feature of sclerosing lymphocytic lobulitis. This is in contrast to most breast disorders where $\mathrm{T}$ cells, and to a lesser 
extent macrophages, are the main inflammatory cells, with only a small number of B cells. ${ }^{6}$ Class II major histocompatibility antigens are usually not expressed in the normal resting mammary epithelium, ${ }^{7}$ although occasionally weak staining may be seen. Strong class II MHC expression in the normal breast is only seen during pregnancy and lactation. The intensity of HLA-DR staining in women with sclerosing lymphocytic lobulitis varies from weak to strong, sometimes within a single biopsy specimen. Thus the patchy epithelial expression of HLA-DR in both cases reported here is consistent with sclerosing lymphocytic lobulitis, but is not strong supportive evidence. The significance of the absence of epithelial staining by LN3 in the series of Tomaszewski $e t$ $a l$ is difficult to judge as they give limited detail of the immunohistochemical method. The association with diabetes mellitus in both of the cases reported previously and one of our cases is another similarity with the disorder in women.

The pattern and immunophenotype of the inflammation, the presence of stromal fibrosis with epithelioid fibroblasts, as well as the association with diabetes mellitus support the view that this disorder in the male breast is the equivalent of sclerosing lymphocytic lobulitis seen in women.

We thank Dr K Eustathios for supplying clinical details for patient 2 .

1 Schwartz IS, Strauchen JA. Lymphocytic mastopathy, An autoimmune disease of the breast? Am 7 Clin Pathol 1990; 93:725-30.

2 Lammie GA, Bobrow LG, Staunton MDM, Levison DA, Page G, Millis RR. Sclerosing lymphocytic lobulitis of the breast - evidence for an autoimmune pathogenesis. Histopathology 1991;19:13-20.

3 Tomaszewski JE, Brooks JSJ, Hicks D, Livolski VA. Diabetic mastopathy: a distinctive clinicopathological entity. Hum mastopathy: a distinctive
Pathol 1992;23:780-6.

4 Ashton MA, Lefkowitz M, Tavassoli FA. Epithelioid stromal cells in lymphocytic mastitis - a source of confusion with invasive carcinoma. Mod Pathol 1994;7:49-54.

5 Epenetos AA, Bobrow LG, Adams TE, Collins CM, Isaacson PG, Bodmer WF. A monoclonal antibody that detects HLA-D region antigen in routinely fixed, wax embedded sections of normal and neoplastic lymphoid tissue. $¥$ Clin Pathol 1985;38:12-17.

6 Giorno R. Mononuclear cells in malignant and benign human breast tissue. Arch Pathol Lab Med 1983;107:41517.

7 Bartek J, Petrek M, Vojtesek B, Bartkova J, Kovarik J Rejthar A. HLA-DR antigens on differentiating mammary gland epithelium and breast tumours. $B r \quad \mathcal{f}$ Cancer gland epithelium

\title{
Association between thyroid cancer of cribriform variant and familial adenomatous polyposis
}

\author{
K Hizawa, M Iida, TYao, K Aoyagi, Y Oohata, R Mibu, KYamasaki, T Hirata, \\ M Fujishima
}

Second Department of

Internal Medicine,

Faculty of Medicine,

Kyushu University,

Maidashi 3-1-1,

Fukuoka 812, Japan

K Hizawa

K Aoyagi

M Fujishima

Second Department of Pathology

TYao

First Department of

Surgery

Y Oohata

R Mibu

Division of Diagnostic

Ultrasound

KYamasaki

T Hirata

Division of

Gastroenterology,

Department of

Medicine,

Kawasaki Medical

School, Kurashiki,

Japan

M Iida

Correspondence to:

Dr Kazuoki Hizawa.

Accepted for publication 12 December 1995

\begin{abstract}
A case of a 20 year old Japanese woman who developed thyroid cancer exhibiting unusual cribriform structures while being followed up for familial adenomatous polyposis/Gardner's syndrome is reported. The patient presented with osteomas, pigmented retinal lesions, and adenomas of the duodenum and the papilla of Vater, in addition to numerous adenomatous polyps in the colorectum. On ultrasonography, the thyroid cancer was localised to the right lobe and was identified as an irregular, internal echo tumour with a peripheral hypoechoic zone, measuring $1.8 \mathrm{~cm}$ in diameter. Histological examination of the resected tumour showed a concomitance of papillary proliferation and cribriform structures with follicles of varying sizes. These features can be distinguished from sporadic thyroid cancer.

(F Clin Pathol 1996;49:611-613)
\end{abstract}

Keywords: familial adenomatous polyposis, thyroid cancer.
Familial adenomatous polyposis (FAP)/ Gardner's syndrome is an inherited disorder caused by a germline mutation of the APC gene. It is characterised by intestinal adenomatous polyps predisposing to cancer. Recent long term, follow up studies have clarified that patients with this condition are also susceptible to malignancies involving multiple sites. ${ }^{12}$ The thyroid gland, particularly in women, is reported to be the most frequently involved organ. ${ }^{2}$ Here, we present a young Japanese woman with thyroid cancer exhibiting unusual histological features of cribriform proliferation. These features are similar to those described recently by Harach et al. ${ }^{3}$ We also review previous cases described in the literature. ${ }^{4-9}$

\section{Case report}

A 20 year old Japanese woman was admitted to hospital in July 1993 for treatment of colonic polyposis. The patient had been diagnosed as having familial adenomatous polyposis at the age of 11 years, when her mother had undergone a total colectomy for colon cancer involving adenomatosis of the colorectum. The patient's condition had been good and she had 